\title{
Development and validation of stability indicating liquid chromatographic (RP-HPLC) method for estimation of ubidecarenone in bulk drug and formulations using quality by design (QBD) approach
}

\author{
Abdul Muheem', Faiyaz Shakeel², Sobiya Zafar', Mohammed Asadullah Jahangir³, \\ Musarrat Husain Warsi', Gaurav Kumar Jain ${ }^{1}$, Farhan Jalees Ahmad ${ }^{1 *}$ \\ ${ }^{I}$ Nano Research Laboratory, Department of Pharmaceutics, School of Pharmaceutical Education \& Research, Jamia \\ Hamdard, New Delhi, India, ${ }^{2}$ Center of Excellence in Biotechnology Research (CEBR), King Saud University, Riyadh, Saudi \\ Arabia, ${ }^{3}$ Faculty of Pharmacy, Glocal University, Saharanpur, India
}

\begin{abstract}
A novel, accurate, precise and economical stability indicating Reverse Phase-High Performance Liquid Chromatography (RP-HPLC) method, was developed and validated for the quantitative determination of ubidecarenone (UDC) in bulk drug, UDC marketed formulation and UDC loaded cubosomes (CBMs) nanocarriers through Response surface methodology (RSM) design with three factors and three levels was performed to optimize the chromatographic variables followed by forced degradation studies of UDC were performed to detect degradation peak. RP-HPLC separation was achieved using mobile phase consisting of Acetonitrile:Tetrahydrofuran:Deionised water in the ratio 55:42:3 and a flow rate of $1.0 \mathrm{~mL} / \mathrm{min}$ was optimized with a standard retention time (Rt) of $2.15 \mathrm{~min}$, through experiment. The method was found linear in the concentration range of $5-100 \mu \mathrm{g} / \mathrm{mL}$ with a regression coefficient of 0.999. The limit of detection (LOD) and limit of quantification (LOQ) were found to be $3.04 \mu \mathrm{g} / \mathrm{mL}$ and $9.11 \mu \mathrm{g} / \mathrm{mL}$, respectively.
\end{abstract}

Keywords: High Performance Liquid Chromatography/validation/stability. Response surface methodology (RSM). Ubidecarnenone (UDC)/evaluation.

\section{INTRODUCTION}

Ubidecarenone (UDC), also known as Coenzyme Q10 or Ubiquinone, is a natural antioxidant found in various plant and animal sources. UDC is a high molecular weight (863.34 g/Mol) hydrophobic drug molecule under BCS class II (Tran et al., 2001) (Figure 1). Absorption of UDC is very complex and associated with active transport mechanisms. UDC is absorbed as chylomicrons in the small intestine and then transported into the lymph to peripheral blood, but due to low aqueous solubility UDC has slow and limited absorption properties (Miles, 2007). Total body contents of UDC in healthy volunteer have been estimated to be 0.5-1.5 gm (Greenberg, Frishman, 1990). When administrated through the oral route, the time $\left(\mathrm{T}_{\max }\right)$

\footnotetext{
*Correspondence: F. J. Ahmad. Nano Research Laboratory, Department of Pharmaceutics, School of Pharmaceutical Education \& Research, Jamia Hamdard, New Delhi, India, 110062. Tel.: +91-09810720387; Fax: +91-11-26059663. E-mail: abdulmuheemkhan@gmail.com
}

of peak plasma concentration $\left(\mathrm{C}_{\max }\right)$ was found to be $\sim 6-8$ $\mathrm{h}$ (Hosoe et al., 2007). The reported prolonged elimination half-life in healthy volunteers following a single dose of deuterated UDC is $\sim 33 \mathrm{~h}$ (Miles, 2007). UDC acts as antioxidant, cardioprotective, nephroprotective, membrane stabilizer and helps in the generation of ATP through the electron transport chain (Flowers, Hartley, Rees, 2013). Reduced form of UDC $\left(\mathrm{UQH}_{2}\right)$ accepts electrons from the oxidized form of UDC (UQ -) but is unable to hold it tightly, eventually donate the electron to another acceptor and thus UDC acts as an antioxidant (Ernster, Dallne, 1995; Kapoor, Kapoor, 2013). National cancer institute (NCI) reported low UDC blood levels in people with myeloma, lymphoma and cancers of the breast, lung, prostate, pancreas, colon, kidney and head and neck. The researchers further suggested that UDC helps the immune system and thus it may be useful as a secondary treatment of cancer.

Quality by design $(\mathrm{QbD})$ Experimental designs are suitable tools to control and optimize the various variables. 


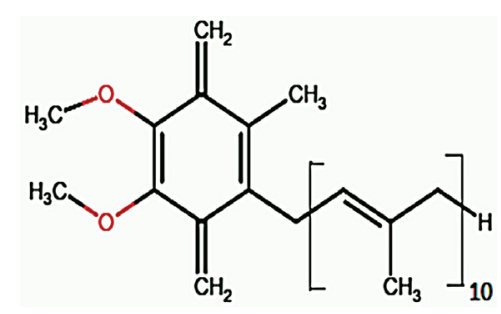

FIGURE 1 - Chemical structure of Ubidecarnenone.

In modern times, 3-level factorial designs which include, central composite design and Box-Behnken design, are being used for the optimization of formulation conditions (Graves et al., 1998). Box-Behnken design is the efficient approach to analyse the various process variables when the interaction between them is very complicated (Box, Behnken, 1960). The utility of Response surface methodology (RSM) for the analytical development and validation has been reported for several drugs e.g. lenalidomide (Hasnain et al., 2013) and amoxicillin trihydrate (Beg et al., 2012), but, no literature is available so far, for the optimized analytical method of UDC using RSM. Various High Performance Liquid Chromatography (HPLC) methods have been developed for the detection of UDC in blood plasma, raw materials and dietary supplements (Graves et al., 1998; Hansen et al., 2004; Lunetta, Roman, 2008). Several other analytical methods such as HPTLC, HPLC, APCI LC-MS and spectroscopic techniques had been previously employed to analyze the stability of UDC (Abdel-Kader et al., 2016; Lang, Packer, 1987; Vadhanavikit et al., 1984). Lang and Parker (1987) reported a reverse phase HPLC (RP-HPLC) method with a step extraction procedure, UV detection, and a run time of about $20 \mathrm{~min}$, for the estimation of UDC in biological fluids, raw materials and dietary supplements (Lang, Parker, 1987). But so far to our present knowledge, there was no RP-HPLC stability-indicating method for assessing UDC in bulk drug and pharmaceutical forms using a UVvisible detector and a direct injection and a run time of $2.15 \mathrm{~min}$. The objective of the present investigation was to develop and validate a novel, simple, rapid, precise, economical and accurate RP-HPLC stability-indicating method for quantification of UDC in bulk drug and pharmaceutical formulation as per ICH guidelines using Design Expert ${ }^{\circledR} 10.0$ software (Stats-Ease) (ICH, 2005).

\section{EXPERIMENTAL}

\section{Material}

Ubidecarenone (UDC) was obtained from Mitsubishi Gas Chemical Company Inc. (Japan). Marketed soft gelatine capsule of UDC (brand name; I.Q 10B) containing $10 \mathrm{mg}$ of UDC was purchased through a local pharmacy. Acetonitrile, tetrahydrofuran, methanol, isopropyl alcohol and deionised water employed were of HPLC grade (Merck, India). Purified water was obtained from Milli-Q water purification system (Millipore) for conducting all the experiments. All other chemicals and reagents were of analytical grade and were purchased by Merck, India.

\section{HPLC equipment and chromatographic conditions}

HPLC analysis was performed on a Shimadzu system comprising of quaternary LC-10A VP pumps, a variable-wavelength programmable UV-visible detector, SPD-10AVP column oven and a SCL 10AVP system controller consisting of $25 \mathrm{~cm} \times 4.6 \mathrm{~mm}$ i.d., $5 \mu \mathrm{m}$ particle, 516 C18 DB reversed phase column (Phenomenex). A variety of mobile phases were investigated in the development of HPLC method suitable for analysis of UDC in the bulk drug. The selection of the mobile phase was based on sensitivity and suitability for drug content estimation. The mobile phase that consisted of acetonitrile (ACN), tetrahydrofuran (THF) and deionised water (DW) in different ratio was used which was filtered through a $0.20 \mu \mathrm{m}$ pore size nylon membrane, degassed ultrasonically and pumped in isocratic mode at a flow rate as optimized using RSM. The Stock solution of UDC was prepared in an accurate volume of methanol $(\mathrm{MeOH})$ and working standard solutions were freshly prepared by diluting the primary stock solutions with mobile phase during the time of analysis. Samples were injected by means of Rheodyne injector and elution of analytes was monitored at a wavelength of $275 \mathrm{~nm}$ (Table I).

TABLE I - HPLC instrument and chromatographic conditions

\begin{tabular}{lc}
\hline Process parameter & Chromatographic conditions \\
\hline Instrument & Shimdazu (LC-10 AT VP) \\
Column & $5 \mu \mathrm{m}$ RP 18 (C18), \\
& Phenomenex® $100,(250 \times 4.6 \mathrm{~mm})$ \\
Mobile phase & ACN: THF: DW \\
Run time & $10 \mathrm{~min}$ \\
Detector & UV- Vis spectrophotometer \\
Detection wavelength & $275 \mathrm{~nm}$ \\
\hline
\end{tabular}

\section{Preparation of calibration curve}

A Standard stock solution of UDC $(1000 \mu \mathrm{g} / \mathbf{m L})$ was prepared by dissolving accurately weighed amount of UDC (10 mg) in an accurate volume of $\mathrm{MeOH}$ in a $10 \mathrm{~mL}$ 
volumetric flask. The volume was made up to $10 \mathrm{~mL}$ with the mobile phase. The prepared solution was stored at $4{ }^{\circ} \mathrm{C}$ away from light. From this stock solution a concentration range of $5-100 \mu \mathrm{g} / \mathrm{mL}$ was prepared by further dilution. These diluted samples were filtered through $0.20 \mu \mathrm{m}$ nylon membrane filter before analysis.

\section{Optimization of analytical variables}

The optimization of analytical variables was carried out using RSM. The mobile phase comprising the mixture of ACN, THF and DW was selected from preliminary screening. In Design Expert ${ }^{\circledR} 10.0$ software, the independent variables- ACN, THF and flow rate were entered with their actual levels. Box-Behnken design of experiments was followed with 3 factors, 2 levels and 17 runs in the experimental design of analytical method (Box, Behnken, 1960).

$\mathrm{Y}_{\mathrm{i}}=\mathrm{b}_{\mathrm{o}}+\mathrm{b}_{1} \mathrm{X}_{1}+\mathrm{b}_{2} \mathrm{X}_{2}+\mathrm{b}_{3} \mathrm{X}_{3}+\mathrm{b}_{12} \mathrm{X}_{1} \mathrm{X}_{2}+\mathrm{b}_{13} \mathrm{X}_{1} \mathrm{X}_{3}+$ $b_{23} X_{2} X_{3}+b_{11} X_{1}^{2}+b_{22} X_{2}^{2}+b_{33} X_{3}^{2}$

where, $Y_{i}$ is the dependent variable; $b_{o}$ is the intercept; $b_{1}$ to $b_{3}$ are the regression coefficients; $X_{1} X_{2}, X_{3}$ are the independent variables that were selected on the basis of pilot experiments. $X_{1}$ acetonitrile, $X_{2}$ tetrahydrofuran and $X_{3}$ is the flow rate, while the dependent variables were retention time (Rt) and peak area, as shown in Table II.

TABLE II - Assigned values of the chromatographic variables with their levels in the response surface methodology

\begin{tabular}{lcc}
\hline \multirow{2}{*}{ Factors } & \multicolumn{2}{c}{ Levels } \\
\cline { 2 - 3 } & Low (-1) & High (+1) \\
\hline Acetonitrile (X1) (\%) & 50 & 60 \\
Tetrahydrofuran (X2) (\%) & 38 & 46 \\
Flow rate (X3) (mL/min) & 0.75 & 1.25 \\
\hline
\end{tabular}

\section{Method validation}

The analytical method was validated with respect to the ICH guidelines Q2 (R1) for parameters such as linearity, accuracy, precision, and specificity, limit of detection (LOD), limit of quantification (LOQ), robustness and ruggedness and forced degradation studies $(\mathrm{ICH}$, 2005).

\section{Linearity and calibration range}

A standard calibration curve of UDC was prepared using ten different concentrations, ranging from 5-100 $\mu \mathrm{g} / \mathrm{mL}$ after serial dilution of stock solution $(1000 \mu \mathrm{g} / \mathrm{mL})$. Each concentration in the series was made each day to determine the linearity of UDC $(n=3)$. The linear calibration curve of peak area versus drug concentration was plotted using the least square regression method and evaluated for linearity and range.

\section{Accuracy as recovery}

The recovery of the method was determined by spiking a previously analyzed test solution with the additional drug standard solution. Accuracy was determined by adding 50,100 and 150\% extra UDC to standard UDC solution $(50 \mu \mathrm{g} / \mathrm{mL})$ to evaluate the mean, standard deviation and relative standard of the peak area to their corresponding drug concentration.

\section{Precision}

The precision of the assay was determined by repeatability (intra-day) and intermediate precision (interday). Repeatability refers to the use of analytical procedure within a laboratory over a short period of time that was evaluated by comparing the assays on different days. Inter- and intra-day variation, analysis of UDC solutions at three different concentrations $(20,40$ and $60 \mu \mathrm{g} / \mathrm{mL})$ were determined in triplicates as per ICH guidelines ( $\mathrm{ICH}$, 2005).

\section{Limit of Detection (LOD) and Limit of Quantification (LOQ)}

Limit of detection and limit of quantification was calculated by the method based on standard deviation of the response for the blank injection in triplicate and the slope (S) of the calibration curve. The peak area of the blank was calculated. The LOD and LOQ were determined using following formulae:

$$
\mathrm{LOD}=3.3 \sigma / \mathrm{S} \quad \mathrm{LOQ}=10 \sigma / \mathrm{S}
$$

Here $\sigma$ is the standard deviation of the blank response and $\mathrm{S}$ is the slope of the calibration curve.

\section{Robustness and ruggedness}

The proposed method was performed by introducing slight changes in the chromatographic conditions such as wavelength for detection $( \pm 2 \mathrm{~nm})$, flow rate $( \pm 0.25 \%)$ and mobile phase composition $( \pm 5 \%)$. The effect in Rt was evaluated individually $(n=3)$. The ruggedness of the method was assessed by comparison of the intra and inter-day assay result of UDC that has been performed by two analysts in the same laboratory. 


\section{Forced degradation studies of UDC}

Forced degradation studies were carried out to provide some information about the drug stability and to validate the specificity of the drug quantification of the assay. The standard solution of UDC was exposed to accelerated degradation by alkaline, acidic, oxidative and direct exposure to sunlight conditions.

\section{Acid and alkaline induced degradation studies}

Two aliquots of UDC standard (10 mg each) were dissolved in methanol in a $10 \mathrm{~mL}$ volumetric flask and volume was made up to $10 \mathrm{~mL}$ with $0.1 \mathrm{M} \mathrm{HCL}$ and $0.1 \mathrm{M}$ $\mathrm{NaOH}$ separately to give strength of $1000 \mu \mathrm{g} / \mathrm{mL}$. The solutions were refluxed over 8 hours on an incubator at 80 ${ }^{\circ} \mathrm{C}$ excluding the possible degradation by light. The resultant solutions were neutralized with $0.1 \mathrm{M} \mathrm{HCL}$ and $0.1 \mathrm{M}$ $\mathrm{NaOH}$. Further the resulting mixtures were diluted with mobile phase, ACN:THF:DW (55:42:3) to give the final concentration of $50 \mu \mathrm{g} / \mathrm{mL}$ and was injected for analysis.

\section{Neutral degradation studies}

To perform the neutral degradation ( $\mathrm{pH}-7), 10 \mathrm{mg}$ of UDC was dissolved in methanol in a $10 \mathrm{~mL}$ volumetric flask to give $1000 \mu \mathrm{g} / \mathrm{mL}$ of concentration. The resultant solution was refluxed and performed in the same manner as in alkaline and acidic degradation studies. The resultant solution was refluxed, diluted with mobile phase to a concentration of $50 \mu \mathrm{g} / \mathrm{mL}$ and was injected for analysis.

\section{Hydrogen peroxide induced degradation studies}

To perform the oxidative degradation, $10 \mathrm{mg}$ of UDC was dissolved in methanol in a $10 \mathrm{~mL}$ volumetric flask and volume was made up to $10 \mathrm{~mL}$ with $3 \%$ hydrogen peroxide to give $1000 \mu \mathrm{g} / \mathrm{mL}$ of concentration. The solutions were refluxed over 8 hours on an incubator at $80^{\circ} \mathrm{C}$. The resultant solution was refluxed, diluted with mobile phase to a concentration of $50 \mu \mathrm{g} / \mathrm{mL}$ and was injected for analysis.

\section{Thermal induced degradation studies}

Ten milligram $(10 \mathrm{mg})$ of UDC were kept in a stability chamber at $100{ }^{\circ} \mathrm{C}$ for $24 \mathrm{hrs}$. The dried sample was dissolved in methanol and the volume made up to 10 mL with ACN:THF:DW (55:42:3). The resultant solution was refluxed over $8 \mathrm{hrs}$ in an incubator at $80^{\circ} \mathrm{C}$ and further diluted with mobile phase to a concentration of $50 \mu \mathrm{g} / \mathrm{mL}$ before injecting for analysis.

\section{Photo induced degradation studies}

For the photolytic degradation study of UDC, the experiment was performed in the presence of UV light $(254 \mathrm{~nm})$ for 24 hours and further exposure to sunlight for one day. $10 \mathrm{mg}$ of light exposed drug was dissolved in a volumetric flask using methanol. The volume was made up to $10 \mathrm{~mL}$ using ACN: THF: DW (55:42:3) as a mobile phase to give $50 \mu \mathrm{g} / \mathrm{mL}$ of concentration and the final solution was injected for analysis.

\section{Analysis of UDC in cubosomes (UDC-CBMs) and marketed dosage form}

UDC-loaded cubosome (UDC-CBMs) were prepared by the fragmentation of glyceryl monooleate (GMO) in PF 127 and water, using modified dilutionsonication method (Spicer et al., 2001). The obtained UDC-GCBMs had particle size in the range of 120-160 $\mathrm{nm}$ (Muheem et al., 2017). UDC-GCBMs equivalent to $10 \mathrm{mg}$ of UDC i.e. $2 \mathrm{~mL}$ of UDC-GCBMs was taken in a $10 \mathrm{~mL}$ volumetric flask and sonicated in an ultrasonic bath for $5 \mathrm{~min}$. The solution was further diluted with the mobile phase. The resultant solution was filtered using $0.2 \mu \mathrm{m}$ nylon membrane filter and injected for analysis.

Twenty capsules (each containing $10 \mathrm{mg}$ UDC) were taken, and the powder was collected and weighed. An accurately weighed portion of the powder equivalent to $10 \mathrm{mg}$ of UDC was taken in $10 \mathrm{~mL}$ volumetric flask. The sample was sonicated in ultrasonic bath for $5 \mathrm{~min}$ and further diluted with mobile phase. The resultant solution was filtered using $0.2 \mu \mathrm{m}$ nylon membrane filter and injected for analysis.

\section{Statistical analysis}

The experiments were carried out in triplicate $(n=3)$ using freshly prepared samples. The results were then expressed as mean, standard deviation (SD) and relative standard deviation (RSD) of the peak areas and their corresponding concentrations were then calculated

\section{RESULTS}

\section{Selection of mobile phase}

The mobile phase was critically selected based on sensitivity, ease of preparation, availability, compatibility for stability studies, the time period for the analysis, the cost of solvents and most importantly the solubility of UDC in the mobile phase. Various mobile phases using different ratios of solvents such as ACN-THF-DW, ACNIPA-DW, THF-IPA-DW and ACN-MeOH-DW were studied for the analysis of UDC. Mobile phase consisting 
of ACN-IPA-DW, THF-IPA-DW and ACN-MeOH-DW did not give any defined sharp peaks and had high Rt (Data not given). A Rt of 2.15 minutes with sharp peaks and no tailing was obtained with ACN: THF: DW.

\section{Experimental design optimization}

To identify the robust and optimized mobile phase composition the positive and/or negative interactions among the independent variables were traded off. The software generated 17 trials, given as coded variables, with the respective data for Rt and peak area, depicted in Table III. The response surfaces and the relationship between the independent variables ACN (X1), THF (X2), and flow rate (X3) on dependent variables, i.e., Rt (Y1) and peak area (Y2) are shown in Figure 2 (A-F). The Rt (Y1) was found to be influenced by the concentration of THF and flow rate. A nonlinear relationship was observed (Figure 2A), where the Rt proportionally decreases at initial levels and then increases with the increasing concentration of THF in the mobile phase mixture. A similar relationship was observed with the flow rate (Figure 2B). The concentration of THF and flow rate was found to influence the response variable (Y2). A curvilinear plot (Figure 2D-F) was obtained indicating a proportionate increase in peak area with increasing concentration of THF and flow rate, whereas with the gradual increase in $\mathrm{ACN}$ in mobile phase, a decrease in peak area was observed. As compared to other models, the classical second degree model with a quadratic experimental domain was suggested best by the design with the highest least squares regression value for response $\mathrm{Y} 1\left(\mathrm{r}^{2}=0.9515\right)$ and $Y 2\left(r^{2}=0.9733\right)$. Linear polynomial equations were studied to better understand the effect of interaction among the independent variables. The linear polynomial equations generated from ANOVA are depicted below:

Retention time $(\mathbf{Y} 1)=+2.14+0.47 \mathrm{X} 1-0.49 \mathrm{X} 2-$

$0.86 \mathrm{X} 3-0.23 \mathrm{X} 1 \mathrm{X} 2-0.31 \mathrm{X} 1 \mathrm{X} 3-0.16 \mathrm{X} 2 \mathrm{X} 3-$

$0.10 \times 1^{2}+0.79 \times 2^{2}+1.34 \times 3^{2}$

Peak Area $(Y 2)=+87561.80-6880 X 1+9701.38 X 2+$ 18958.88X3 - 1171.0X1X2+3113.50X1X3+ $2499.75 \times 2 \times 3+4963.47 \times 1^{2}+1705.22 \times 2^{2}+$ $7720.23 \times 3^{2}$

From the polynomial equations, it has been observed that all three independent variables with a positive
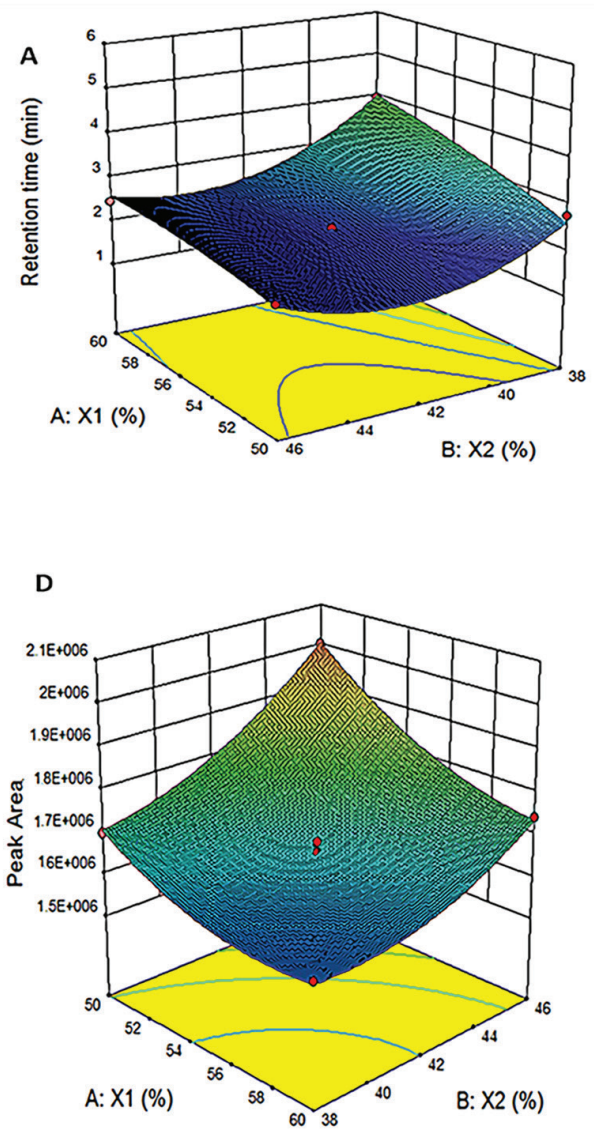
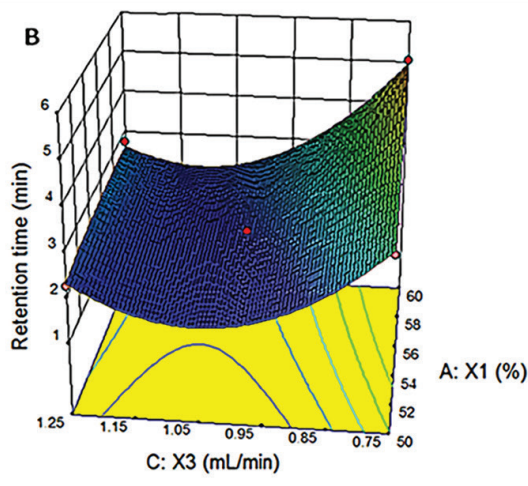

E

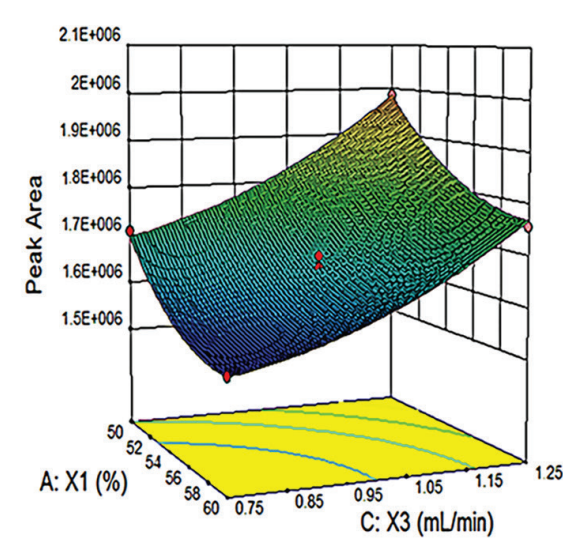

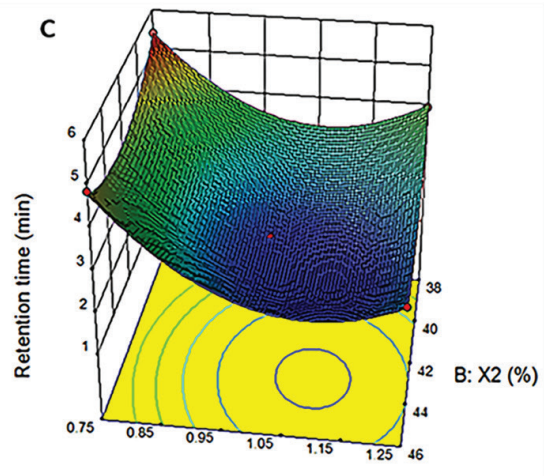

C: $X 3(\mathrm{~mL} / \mathrm{min})$

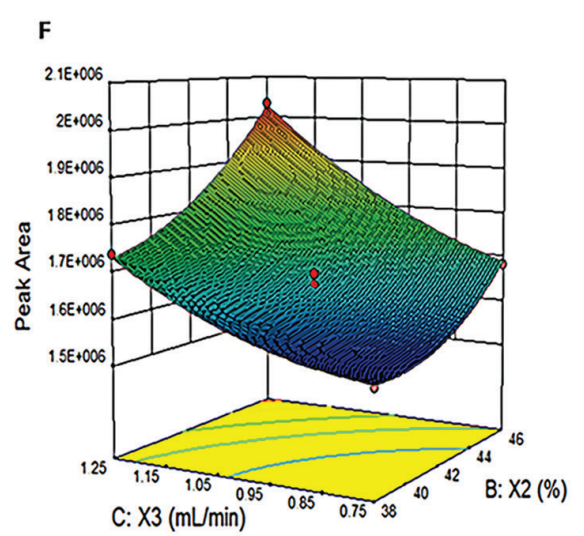

FIGURE 2 - 3D-Response surface plots of (A) retention time and (B) Peak area for UDC 
sign indicates a positive effect on both the observed responses and negative sign showed a negative effect on both the observed responses. However, for Rt (Y1), the independent variable ACN (X1) has a positively influence on the Rt while the variable X2 and X3 favors the smaller value. However, the peak area (Y2) was found to be decreased with the variable $\mathrm{X} 1$ while a greater influence of X2 and X3 was observed.

TABLE III - Experimental observed response in Box-Behnken design used for UDC method development

\begin{tabular}{lccccc}
\hline Trial & $\mathbf{X 1}$ & $\mathbf{X 2}$ & $\mathbf{X 3}$ & $\mathbf{Y 1}$ & $\mathbf{Y 2}$ \\
\hline 1 & 55 & 38 & 0.75 & 5.42 & $15,25,354$ \\
2 & 50 & 42 & 1.25 & 2.29 & $19,86,893$ \\
3 & 60 & 38 & 1 & 3.96 & $15,85,297$ \\
4 & 55 & 46 & 1.25 & 2.78 & $20,34,394$ \\
5 & 55 & 42 & 1 & 2.15 & $16,79,545$ \\
6 & 60 & 46 & 1 & 2.45 & $17,42,918$ \\
7 & 55 & 38 & 1.25 & 4.12 & $17,37,383$ \\
8 & 50 & 46 & 1 & 2.14 & $20,02,541$ \\
9 & 55 & 42 & 1 & 2.15 & $16,43,424$ \\
10 & 60 & 42 & 1.25 & 2.78 & $17,57,359$ \\
11 & 50 & 42 & 0.75 & 3.36 & $17,09,838$ \\
12 & 50 & 38 & 1 & 2.75 & $16,98,284$ \\
13 & 55 & 42 & 1 & 2.14 & $16,55,362$ \\
14 & 55 & 46 & 0.75 & 4.86 & $16,82,379$ \\
15 & 60 & 42 & 0.75 & 5.07 & $15,18,751$ \\
16 & 55 & 42 & 1 & 2.15 & $16,44,395$ \\
17 & 55 & 42 & 1 & 2.15 & $16,45,384$ \\
\hline$X 1$,
\end{tabular}

$\mathrm{X} 1$, Acetonitrile (\%); X2, tetrahydrofuran (\%); X3, flow rate (mL/min); Y1, retention time (minute); Y2, Peak area.

\section{Method validation}

\section{System suitability}

The $\%$ CV of peak area and Rt for UDC was found to be within $2 \%$, indicating the suitability of the system. The number of theoretical plates and USP tailing factor for the six replicate injections were found to be $9265.5 \pm$ $1.5 \%$ and $1.38 \pm 0.3 \%$ respectively.

\section{Linearity and calibration range}

The standard calibration plot demonstrated a good relationship between concentration of UDC and area of the chromatogram over a concentration range of $5-100 \mu \mathrm{g} / \mathrm{mL}$. The linear regression equation was $y=12589 x-26462$ with the regression coefficient of 0.999 . The LOD and LOQ of the developed method were found to be 3.04 and 9.11 $\mu \mathrm{g} / \mathrm{mL}$, respectively, which showed that the method was sensitive enough to detect and quantify UDC. Table IV summarizes the statistical data $(\mathrm{n}=3)$ for regression analysis.

TABLE IV - Summary of linear regression data for the calibration plot $(n=6)$ obtained from design of expert based design matrix

\begin{tabular}{lc}
\hline Statistical parameters & HPLC Values \\
\hline Linearity range $(\mu \mathrm{g} / \mathrm{mL})$ & $5-100$ \\
Regressed equation & $\mathrm{Y}=12589 \mathrm{X}-26462$ \\
Correlation coefficient $\left(\mathrm{R}^{2}\right)$ & 0.999 \\
Slope $\pm \mathrm{SD}$ & $12589 \pm 102.320$ \\
Intercept $\pm \mathrm{SD}$ & $26462 \pm 25.015$ \\
Limit of detection $(\mu \mathrm{g} / \mathrm{mL})$ & 3.04 \\
Limit of quantification $(\mu \mathrm{g} / \mathrm{mL})$ & 9.11 \\
\hline
\end{tabular}

\section{Accuracy and precision}

The percentage recovery was found to be in the range of $99.94-102.29 \pm 1.36 \%$. The $\%$ RSD for recovery value is less than 2 , thus satisfying the acceptance criteria (Table V). The \% RSD for intra-day precision and for inter-day precision was found to be in the range of 0.39-0.9 and 0.6-1.73, respectively. The developed method showed good precision and reproducibility with \% RSD less than 2, as listed in Table VI.

TABLE V - Accuracy study data of the developed UDC HPLC method ( $\mathrm{n}=3$ )

\begin{tabular}{lcccc}
\hline $\begin{array}{l}\text { Excess drug added to } \\
\text { analyte }(\%)\end{array}$ & $\begin{array}{c}\text { Theoretical content } \\
(\mu \mathrm{g} / \mathrm{mL})\end{array}$ & $\begin{array}{c}\text { Concentration found } \\
(\mu \mathrm{g} / \mathrm{mL}) \pm \mathrm{SD}\end{array}$ & \% Recovery & \% RSD \\
\hline 0 & 50 & $49.89 \pm 0.19$ & 99.78 & 0.380 \\
50 & 75 & $75.25 \pm 0.24$ & 100.33 & 0.318 \\
100 & 100 & $100.13 \pm 0.27$ & 100.13 & 0.269 \\
150 & 125 & $125.53 \pm 0.89$ & 100.42 & 0.708 \\
Cubosome formulation & 50 & $49.43 \pm 0.43$ & 98.86 & 0.869 \\
Marketed formulation & 50 & $49.29 \pm 0.74$ & 98.58 & 1.50 \\
\hline
\end{tabular}


Development and validation of stability indicating liquid chromatographic (RP-HPLC) method

TABLE VI - Intra-day and inter-day precision study data of developed UDC HPLC method ( $n=3$ )

\begin{tabular}{lcccc}
\hline $\begin{array}{l}\text { Concentration } \\
(\boldsymbol{\mu g} / \mathbf{m l})\end{array}$ & \multicolumn{2}{c}{ Repeatability (intra-day) } & \multicolumn{2}{c}{ Intermediate precision (inter-day) } \\
\cline { 2 - 5 } & $\begin{array}{c}\text { Mean recovery } \\
\boldsymbol{\mu} \mathbf{g} / \mathbf{m L}) \pm \mathbf{S D}\end{array}$ & $\mathbf{\% ~ R S D}$ & $\begin{array}{c}\text { Mean recovery } \\
(\boldsymbol{\mu g} / \mathbf{m L}) \pm \mathbf{S D}\end{array}$ & \% RSD \\
\hline 20 & $19.2 \pm \mathbf{0 . 1 0}$ & 0.520 & $19.6 \pm 0.12$ & 0.612 \\
40 & $38.7 \pm \mathbf{0 . 3 5}$ & 0.904 & $39.5 \pm 0.29$ & 0.734 \\
60 & $58.6 \pm \mathbf{0 . 2 3}$ & 0.392 & $59.3 \pm 0.61$ & 1.02 \\
\hline \multicolumn{4}{c}{ Reproducibility of the developed UDC method } \\
\hline 40 & $18.9 \pm 0.09$ & 0.476 & $19.7 \pm 0.23$ & 1.16 \\
60 & $39.7 \pm 0.23$ & 0.579 & $39.1 \pm 0.44$ & 1.12 \\
\hline
\end{tabular}

Robustness

With small deliberate changes in chromatographic conditions no significant effect on Rt and peak area was observed. The results are presented in Table VII along with system suitability parameters of normal methodology. The influence of each variable on Rt of UDC was insignificant with $\%$ RSD $>2 \%$ implying the robustness of the method.

TABLE VII - Robustness study data of developed UDC HPLC method at different conditions $(\mathrm{n}=3$ )

\begin{tabular}{lccc}
\hline $\begin{array}{l}\text { Chromatographic } \\
\text { conditions }\end{array}$ & $\begin{array}{c}\text { Retention } \\
\text { time }\end{array}$ & \% RSD & $\begin{array}{c}\text { Optimized } \\
\text { value }\end{array}$ \\
\hline $\begin{array}{l}\text { Wavelength of } \\
\text { detection (nm) }\end{array}$ & & & \\
274 & 2.17 & 0.46 & 275 \\
275 & 2.15 & 0.27 & \\
276 & 2.15 & 0.27 & \\
Flow rate (mL/min) & & & \\
$\quad 0.98$ & 2.18 & 0.55 & 1.0 \\
1.0 & 2.16 & 0.37 & \\
1.02 & 2.19 & 0.36 & \\
\% Deionised water & & & \\
2.0 & 2.15 & 0.74 & 3.0 \\
3.0 & 2.15 & 0.51 & \\
4.0 & 2.20 & 0.45 & \\
\hline
\end{tabular}

\section{Forced degradation studies}

To study the forced degradation, the UDC sample was exposed to $0.1 \mathrm{M} \mathrm{HCl}, 0.1 \mathrm{M} \mathrm{NaOH}$, neutral, $3 \%$ $\mathrm{H}_{2} \mathrm{O}_{2}$, dry heat and UV light. The degradation peaks obtained were well distinguished from the parent peak. There were no significant changes in the Rt of UDC, but changes in peak areas were observed due to degradation metabolites eluted at different time points. The sample subjected to acidic stress showed a parent peak at 2.15 min but a slight decrease in the area due to degradation of the parent drug in acidic condition was observed. In acidic stress condition the Rt of the metabolite peak was found to be 2.128 minutes with a $\%$ recovery of $95.63 \%$ (Figure 3-(1) A). UDC sample kept under alkaline stress condition showed an intense metabolite peak at 1.994 minutes, as the sample was highly unstable under alkaline stress condition, the recovery decreased to $44.05 \%$ (Figure 3-(1) B). UDC sample kept in the oven at $100^{\circ} \mathrm{C}$ for 24 hours, upon dilution with subsequent injection showed the peak of parent drug at Rt of 2.18 min with recovery of $98.47 \%$ as shown in Figure 3-(2) (E). UDC sample was found to be stable under neutral condition with \% recovery 81.32 $\pm 0.63 \%$ (Figure 3 -(1) C). The UDC sample was found to be unstable under oxidative stresses with \% recovery of $51.08 \%$ (Figure 3-(2) D). Rt of the parent as well as degradation metabolites with recoveries are summarized in Table VIII and Figure 3 (A-F). UDC is degraded in presence of light. UDC sample kept under photolytic condition showed two peak at 4.718 and 8.581. which depicted the degradation profile of UDC in Figure 3-2 (F).

\section{Analysis of UDC in cubosomes (UDC-CBMs) and marketed dosage form}

The Rt for UDC in bulk drug, CBMs formulation and marketed dosage form was found to be 2.158, 2.156, 2.179 , respectively, confirming the stability of UDC in the formulation (Figure 4A-C). Furthermore, a single parent peak obtained in the analysis of CBMs confirms the fact that no excipient interaction occurred in the formulation.

\section{DISCUSSION}

The method was optimized by the design of 

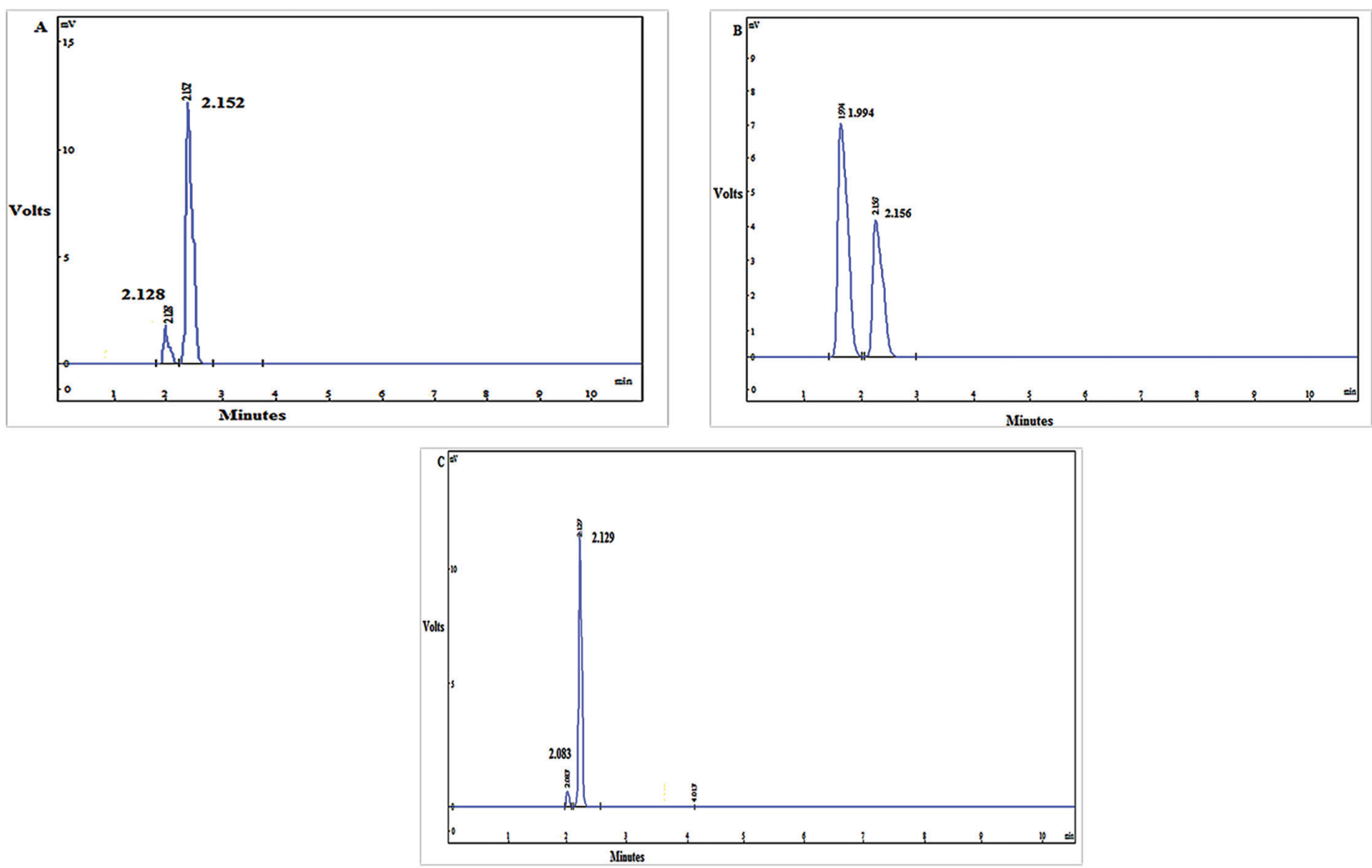

FIGURE 3-(1) - Forced degradation chromatograms of UDC (A) acidic stress; (B) alkaline stress; (C) Neutral stress.
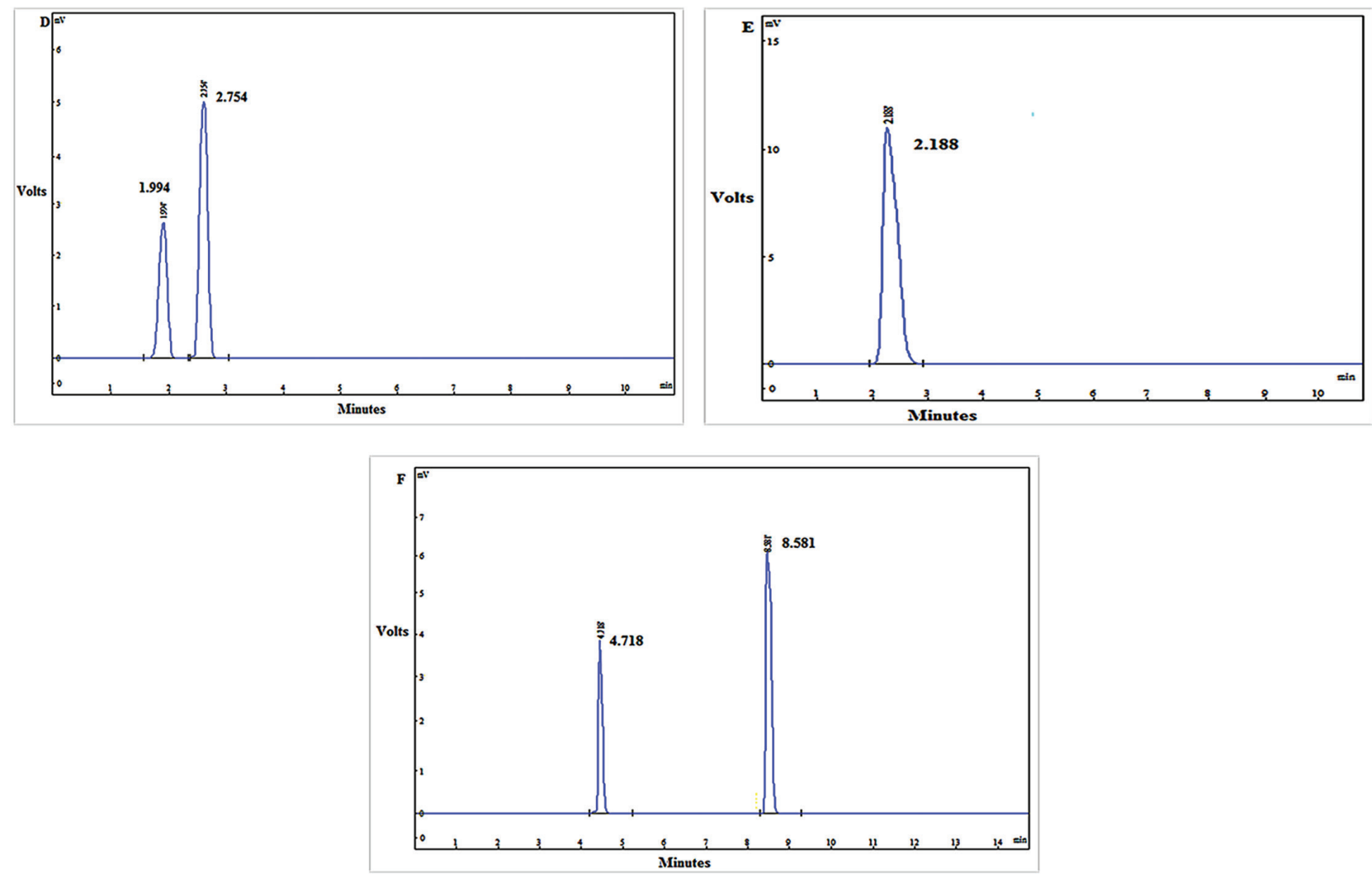

FIGURE 3-(2) - Forced degradation chromatograms of UDC (D) oxidation stress; (E) thermal stress; and (F) photolytic stress. 
TABLE VIII - Forced degradation studies data of UDC at various stress conditions

\begin{tabular}{lccccc}
\hline $\begin{array}{l}\text { Degradation } \\
\text { condition }\end{array}$ & $\begin{array}{c}\text { Rt of UDC after } \\
\text { exposure }(\mathbf{m i n} \pm \text { SD) }\end{array}$ & \%RSD & $\begin{array}{c}\text { Rt of degradation } \\
\text { products (min } \pm \text { SD) }\end{array}$ & \%RSD & $\begin{array}{c}\text { \% Recovery after } \\
\text { 24 hours }\end{array}$ \\
\hline Acidic & 2.152 & 0.465 & 2.128 & 0.575 & $95.63 \pm 0.24$ \\
Alkaline & 2.150 & 0.189 & 1.998 & 0.466 & $44.05 \pm 0.57$ \\
Neutral & 2.129 & 0.697 & 2.083 & 0.669 & $81.32 \pm 0.63$ \\
Oxidative & 2.754 & 0.342 & 1.994 & 0.549 & $53.08 \pm 0.58$ \\
Thermal & 2.188 & 0.509 & - & - & $98.47 \pm 0.19$ \\
Photolytic & 8.581 & 0.165 & 4.778 & 0.116 & $35.14 \pm 0.76$ \\
\hline
\end{tabular}
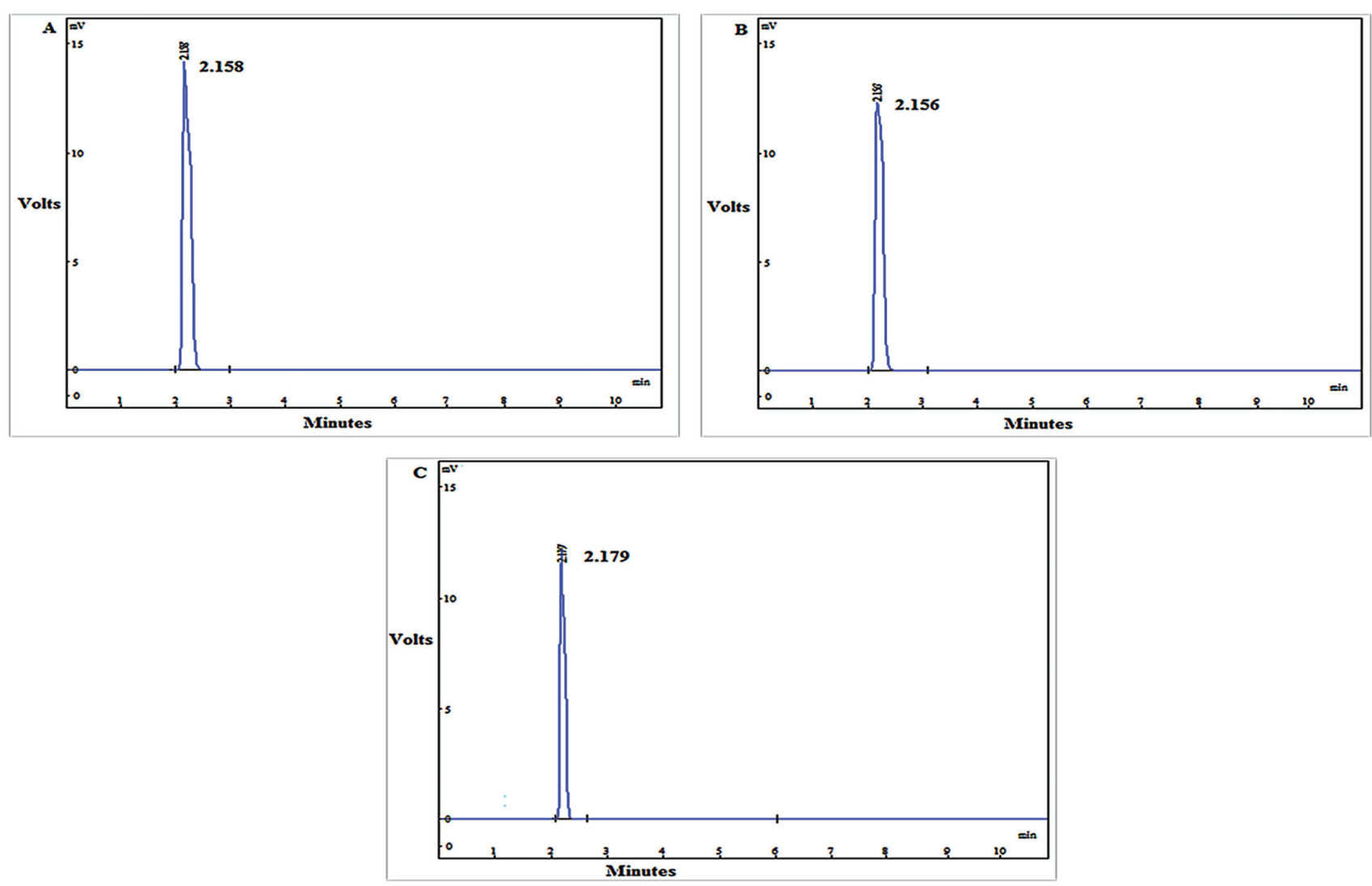

FIGURE 4 - HPLC chromatograms of UDC (A); UDC API (B); UDC peak in cubosome (C); and UDC peak in marketed dosage form.

experimental methodology which also furnished thoughtfully-thorough understanding of the factorresponse relationship and underlying interactions among them. The search for optimum condition was carried out through numerical optimization by 'trading off' various factors to attain the desired goals, that is maximization of peak area and theoretical plates and minimization of Rt and peak tailing to obtain desirability function closer to 1 . The optimized condition obtained was, the mobile phase composition containing ACN (55\%), THF $(42 \%)$ and DW (3\%) and flow rate $(1 \mathrm{~mL} / \mathrm{min})$. The graphical optimization also yielded the optimum solution demarcate within the analytical design space as shown in the chromatogram of UDC (Figure 4A). A Rt of 2.15 min was observed with a reasonable peak area within the defined limits. The analysis of the system suitability test demonstrated that the developed chromatographic conditions can be exploited to provide accurate data. The method was found to be precise (both intra-day and inter-day precision) and the results were found to be consistent with the repeatability of the method for the analysis of UDC in developed formulation. The obtained results for $\%$ recovery of UDC reference substance from formulation were found to be within the acceptable range, 
thus providing evidence that the proposed method was accurate. The result of robustness study showed that no significant effect was observed on Rt and area, with small deliberate changes in chromatographic conditions. There was no significant change in the Rt of UDC, but decrease in area was observed due to degradation metabolites eluted at different time points. The degradation peaks obtained were distinguishable from the parent peak. The method was highly advantageous vis-a-vis in terms of time, economy for determination of UDC formed during stress conditions, as evident from low Rt. The developed method was found to be sensitive which was evaluated in terms of LOD and LOQ. Further, the Rt of UDC in all the dosage forms was similar with respect to the standard UDC without any significant difference in the standard solution. Other parameters, like theoretical plates and peak tailing were found to be within the acceptable limits. Thus the developed method is a corroborated high degree of utility and can be exploited for the routine estimation of UDC in pharmaceutical formulations.

\section{CONCLUSION}

The method was optimized by design of experiment optimization technique using different variables. The measured signal was found to be precise, accurate and linear over the concentration range tested with a correlation coefficient of 0.999 . Moreover, the lower solvent consumption along with the short analytical retention time of $2.15 \mathrm{~min}$ supports a cost effective and environmentally friendly chromatographic procedure. The forced degradation studies provided well distinguishable peaks of parent and degradation metabolites. Thus, the above validated method proved to be a rapid, selective, specific and accurate method which can be exploited for the separation of impurities and quantitative determination of UDC in API and pharmaceutical formulations. The corroborated method supports a high degree of practical utility for estimation of UDC in pharmaceutical dosage forms.

\section{ACKNOWLEDGEMENT}

The work was supported by University Grants Commission (UGC-2012864), India. The authors are also thankful to Dr. Zrien Naz, Dr. Iqbal Ahmad and Dr. Mohammed Anwar, Department of Pharmaceutics, School of Pharmaceutical Education \& Research, Jamia Hamdard, New Delhi, for their linguistic support.

\section{CONFLICT OF INTERESTS}

The authors confirm that this paper's content has no conflict of interests.

\section{REFERENCES}

Abdel-Kader MS, Alam P, Alqasoumi SI. Densitometric HPTLC method for qualitative, quantitative analysis and stability study of coenzyme Q10 in Pharmaceutical formulations utilizing normal and reversed-phase silica gel plates. Pak J Pharm Sci. 2016;29(2):477-484.

Beg S, Kohli K, Swain S, Hasnain S. Development and validation of RP-HPLC method for quantitation of amoxicillin trihydrate in bulk and pharmaceutical formulations using BoxBehnken experimental design. J Liq Chromatogr Relat Technol. 2012;35(3):393-6.

Box GE, Behnken DW. Some new three level designs for the study of quantitative variables. Technomet. 1960;2(4):455-475.

Ernster L, Dallne G. Biochemical, physiological and medical aspects of ubiquinone function. Biochim Biophys Acta (BBA)Mol Basis Dis. 1995;1271(1):195-204.

Flowers N, Hartley L, Rees K. Co-enzyme Q10 supplementation for the primary pre-vention of cardiovascular disease. Cochrane Database Syst Rev. 2013;2:CD010405.

Graves S, Sikorska M, Borowy-Borowski H, Ho RJH, Bui T, Woodhouse C. Analysis of coenzyme Q 10 content in human plasma and other biological samples. Meth Mol Bio. 1998;108:353-365.

Greenberg S, Frishman WHJ. Co-enzyme Q10: a new drug for cardiovascular disease. J Clin Pharmacol. 1990;30(7):596-608.

Hansen G, Christensen P, Tüchsen E, Lund T. Sensitive and selective analysis of coenzyme $\mathrm{Q} 10$ in human serum by negative APCI LC-MS. Analyst. 2004;129(1):45-50.

Hasnain MS, Rao S, Singh MK, Vig N, Gupta A, Ansari A, Sen P, Joshi P, Ansari SA. Development and validation of LC-MS/ MS method for the quantitation of lenalidomide in human plasma using Box-Behnken experimental design. Analyst. 2013;138(5):1581-1588. 
Hosoe K, Kitano M, Kishida H, Kubo H, Fujji K, Kitahara M. Study on safety and bioavailability of ubiquinol (Kaneka $\mathrm{QH}^{\mathrm{TM}}$ ) after single and 4-week multiple oral administration to healthy volunteers. Regul Toxicol Pharmacol. 2007;47(1):19-28.

International Conference on Harmonisation. Guideline. ICH. Validation of analytical procedures: text and methodology. Q2 (R1). Geneva: ICH;2005. 17 p.

Kapoor P, Kapoor A. Coenzyme Q10-A novel molecule. J Ind Acad Clin Med. 2013;14(1):37-45.

Lang JK, Packer L. Quantitative determination of vitamin E and oxidized and reduced coenzyme $\mathrm{Q}$ by high-performance liquid chromatography with in-line ultraviolet and electrochemical detection. J Chrom A. 1987;385:109-117.

Lunetta S, Roman M. Determination of coenzyme Q10 content in raw materials and dietary supplements by high-performance liquid chromatography-UV: collaborative study. J AOAC Int. 2008;91(4):702-708.

Miles MV. The uptake and distribution of coenzyme Q (10). Mitochondrion. 2007;7(Supp 1):S72-S77.
Muheem A, Shakeel F, Warsi MH, Jain GK, Ahmad FJ. A combinatorial statistical design approach to optimize the nanostructured cubosomal carrier system for oral delivery of ubidecarenone for management of doxorubicin induced cardiotoxicity: In Vitro-In Vivo investigations. J Pharm Sci. 2017;106:1-55.

Spicer PT, Hayden KL, Lynch ML, Ofori-Boateng A, Burns JL. Novel process for producing cubic liquid crystalline nanoparticles (cubosomes). Langmuir. 2001;17(19):5748-5756.

Tran MT, Mitchell TM, Kennedy DT, Giles JT. Role of coenzyme Q10 in chronic heart failure, angina, and hypertension. Pharmacother J Human Pharmacol Drug Ther. 2001;21(7):797806.

Vadhanavikit S, Sakamoto N, Ashida N, Kishi T, Folkers K. Quantitative determination of coenzyme Q10 in human blood for clinical studies. Anal Biochem. 1984;142(1):155-158.

Received for publication on $25^{\text {th }}$ May 2017 Accepted for publication on $26^{\text {th }}$ June 2017 